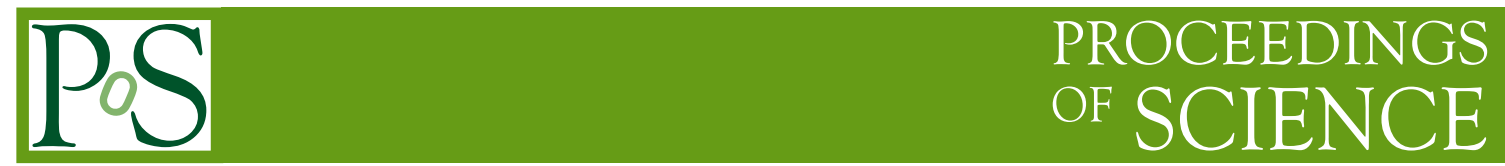

\title{
Charged lepton: results and future prospects
}

\author{
Ryu SAWADA* \\ ICEPP, the University of Tokyo \\ E-mail: sawadadicepp.s.u-tokyo.ac.jp
}

The flavor is not conserved in the quark and the neutrino sectors in the standard model of the particle physics. On the other hand, flavor violation is strictly forbidden in the charged lepton sector. Charged-lepton flavor violation (cLFV) is predicted in the most of new physics models. Searches of cLFV are powerful tools to discover an unambiguous evidence of the new physics. There is a wide variety of experiments searching for cLFV with using charged leptons. In this paper, the latest search results and prospects for the future are discussed.

38th International Conference on High Energy Physics 3-10 August 2016

Chicago, USA

${ }^{*}$ Speaker. 


\section{Introduction}

The standard model (SM) of the fundamental particle physics is a very successful theory. There are however several reasons to expect new undiscovered physics existing at higher energy levels. For example, the SM does not have a particle which can be a candidate of dark matter. There are many new models, for example, supersymmetric grand unified theories, extra-dimension models etc., which solve such problems. The flavor is not conserved in the quark and neutrino sectors; flavor changes in these sectors were confirmed by many experiments. On the other hand, the flavor violation in the charged lepton sector is forbidden in the SM and no charged flavor violation (cLFV) have been observed. The loop contribution of neutrino oscillations is too small to detect, e.g. $\operatorname{Br}(\mu \rightarrow \mathrm{e} \gamma)<10^{-54}$. New physics models beyond the SM largely predict undiscovered heavy particles. In many such theories, predicted branching ratios of cLFV through the flavor mixing of the new particles are sizable $\left(\operatorname{Br}(\mu \rightarrow \mathrm{e} \gamma) \sim 10^{-12}-10^{-14}\right)$.

Several anomalies related to the charged lepton flavor have been reported in various experiments. The result of the muon anomalous magnetic dipole moment measurement by the BNL E821 experiment [1] is approximately $3 \sigma$ away from the SM prediction. The difference could be due to a loop effect where new particles are involved. The correlation between the strength of the cLFV muon decay signals and g-2 must be strong because cLFV decays could happen with processes with additional flavor changing of the new particles causing the effects. It was reported that a measured charge radius of protons with using muonic hydrogen is smaller by $7 \sigma$ [2] from the average value of measurements by electron proton scattering experiments. The reason of the difference is still being discussed; but that may suggest a flavor non-universal effect of new physics. A $3.9 \sigma$ difference from the $\mathrm{SM}$ was observed in the ratio of $\operatorname{Br}\left(\overline{\mathrm{B}}^{0} \rightarrow \mathrm{D}^{*+} \tau^{-} \bar{v}_{\tau}\right)$ and $\operatorname{Br}\left(\overline{\mathrm{B}}^{0} \rightarrow \mathrm{D}^{*+} \mu^{-} \bar{v}_{\mu}\right)$ [3], which may suggest the lepton non-universality. And there are several anomalies in the $b \rightarrow s$ transition, $\frac{\mathrm{Br}\left(\mathrm{B}^{+} \rightarrow \mathrm{K}^{+} \mu^{+} \mu^{-}\right)}{\mathrm{Br}\left(\mathrm{B}^{+} \rightarrow \mathrm{K}^{+} \mathrm{e}^{+} \mathrm{e}^{-}\right)}$[4], $\mathrm{Br}\left(\mathrm{B}_{\mathrm{s}} \rightarrow \phi \mu^{+} \mu^{-}\right)$[5] and $\mathrm{B}^{0} \rightarrow \mathrm{K}^{* 0} \mu^{+} \mu^{-}$angular analysis [6], in which muon pairs are in the final states. Slightly positive excesses of the LFV Higgs decay $h \rightarrow \mu \tau$ events were observed in the CMS Run1 data with a $2.4 \sigma$ significance [7].

In case that the dipole interaction is dominant for cLFV processes, the ratios of the predicted branching ratios of $\mu^{+} \rightarrow \mathrm{e}^{+} \gamma, \mu^{+} \rightarrow \mathrm{e}^{+} \mathrm{e}^{-} \mathrm{e}^{+}$and $\mu \rightarrow \mathrm{e}$ conversion in an aluminum target are $1: \frac{1}{170}: \frac{1}{390}$. The branching ratios of $\tau$ LFV decays are predicted to be $2-5$ orders of magnitude higher than those of muon decays. Predicted branching ratios of cLFV decays in various new physics models are different as shown in Table 1 because the dominating interactions are different. For example $\tau^{-} \rightarrow \mu^{-} \mu^{+} \mu^{-}, \mu^{+} \rightarrow \mathrm{e}^{+} \mathrm{e}^{-} \mathrm{e}^{+}, \mu \rightarrow \mathrm{e}$ conversion etc. are enhanced when the contribution of the four-fermion contact operator is large compared to the dipole-type operator. It is therefore possible to discriminate new physics models with measuring several cLFV channels.

\section{2. cLFV search experiments}

The search of cLFV has been done with rare decay channels of leptons $(\mu, \tau)$, mesons $(\mathrm{K}, \mathrm{J} / \psi$ etc.) and bosons (Z, Higgs). The current limits are summarized in Table 2. Among of them, cLFV searches through muon rare decays, $\mu^{+} \rightarrow \mathrm{e}^{+} \gamma, \mu^{+} \rightarrow \mathrm{e}^{+} \mathrm{e}^{-} \mathrm{e}^{+}$and $\mu \rightarrow \mathrm{e}$ conversion in nuclei, have high sensitivities for new physics thanks to very strong muon sources and their clean signals. Figure 1 shows the obtained $\mu^{+} \rightarrow \mathrm{e}^{+} \gamma$ equivalent upper limits on branching ratios of LFV muon 
Table 1: Predicted ratios of cLFV branching ratios. The numbers are taken from [8].

\begin{tabular}{|c|c|c|c|}
\hline ratio & LHT & MSSM (dipole) & MSSM (Higgs) \\
\hline$\frac{\operatorname{Br}\left(\mu^{-} \rightarrow \mathrm{e}^{-} \mathrm{e}^{+} \mathrm{e}^{-}\right)}{B r(\mu \rightarrow \mathrm{e} \gamma)}$ & $0.02 \ldots 1$ & $\sim 6 \cdot 10^{-3}$ & $\sim 6 \cdot 10^{-3}$ \\
\hline$\frac{B r\left(\tau^{-} \rightarrow \mathrm{e}^{-} \mathrm{e}^{+} \mathrm{e}^{-}\right)}{B r(\tau \rightarrow \mathrm{e} \gamma)}$ & $0.04 \ldots 0.4$ & $\sim 1 \cdot 10^{-2}$ & $\sim 1 \cdot 10^{-2}$ \\
\hline$\frac{B r\left(\tau^{-} \rightarrow \mu^{-} \mu^{+} \mu^{-}\right)}{B r(\tau \rightarrow \mu \gamma)}$ & $0.04 \ldots 0.4$ & $\sim 2 \cdot 10^{-3}$ & $\sim 0.06 \ldots 0.1$ \\
\hline$\frac{B r\left(\tau^{-} \rightarrow \mathrm{e}^{-} \mu^{+} \mu^{-}\right)}{B r(\tau \rightarrow \mathrm{e} \gamma)}$ & $0.04 \ldots 0.3$ & $\sim 2 \cdot 10^{-3}$ & $0.02 \ldots 0.04$ \\
\hline$\frac{\operatorname{Br}\left(\tau^{-} \rightarrow \mu^{-} \mathrm{e}^{+} \mathrm{e}^{-}\right)}{B r(\tau \rightarrow \mu \gamma)}$ & $0.04 \ldots 0.3$ & $\sim 1 \cdot 10^{-2}$ & $\sim 1 \cdot 10^{-2}$ \\
\hline$\frac{B r\left(\tau^{-} \rightarrow \mathrm{e}^{-} \mathrm{e}^{+} \mathrm{e}^{-}\right)}{\left.B r\left(\tau^{-} \rightarrow \mathrm{e}^{-} \mu^{+} \mu^{-}\right)\right)}$ & $0.8 \ldots 2.0$ & $\sim 5$ & $0.3 \ldots 0.5$ \\
\hline$\frac{B r\left(\tau^{-} \rightarrow \mu^{-} \mu^{+} \mu^{-}\right)}{\operatorname{Br}\left(\tau^{-} \rightarrow \mu^{-} \mathrm{e}^{+} \mathrm{e}^{-}\right)}$ & $0.7 \ldots 1.6$ & $\sim 0.2$ & $5 \ldots 10$ \\
\hline$\frac{R(\mu \mathrm{Ti} \rightarrow \mathrm{eTi})}{B r(\mu \rightarrow \mathrm{e} \gamma)}$ & $10^{-3} \ldots 10^{2}$ & $\sim 5 \cdot 10^{-3}$ & $0.08 \ldots 0.15$ \\
\hline
\end{tabular}

decays. The upper limits have been improved as the muon source became stronger from cosmicray muons, muons from stopped pions, then to the muon beams produced by high power proton accelerators.

Table 2: Current upper limits of cLFV decays.

\begin{tabular}{l|r}
\hline Process & Upper limit \\
\hline \hline$\mu^{+} \rightarrow \mathrm{e}^{+} \gamma$ & $4.2 \times 10^{-13}[9]$ \\
$\mu^{+} \rightarrow \mathrm{e}^{+} \mathrm{e}^{-} \mathrm{e}^{+}$ & $1.0 \times 10^{-12}[10]$ \\
$\mu^{-} \mathrm{Au} \rightarrow \mathrm{e}^{-} \mathrm{Au}$ & $7 \times 10^{-13}[11]$ \\
$\tau^{ \pm} \rightarrow \mathrm{e}^{ \pm} \gamma$ & $3.3 \times 10^{-8}[12]$ \\
$\tau^{ \pm} \rightarrow \mu^{ \pm} \gamma$ & $4.4 \times 10^{-8}[12]$ \\
$\tau^{-} \rightarrow 1^{-} 1^{+} 1^{-}$ & $1.5-2.7 \times 10^{-8}[13]$ \\
$\mathrm{K}_{\mathrm{L}}^{0} \rightarrow \mu^{ \pm} \mathrm{e}^{\mp}$ & $4.7 \times 10^{-12}[14]$ \\
$\mathrm{Z} \rightarrow e^{ \pm} \mu^{\mp}$ & $7.5 \times 10^{-7}[15]$ \\
$\mathrm{h} \rightarrow \mu^{ \pm} \tau^{\mp}$ & $1.2 \times 10^{-2}[16]$ \\
\hline
\end{tabular}

\subsection{MEG and MEG II}

The MEG experiment was carried out at Paul Scherrer Institute (PSI) in Switzerland where the most intense DC muon beam up to $10^{8} \mu / \mathrm{sec}$ is available. Figure 2 shows the MEG detector. The high intensity muon beam was stopped on a thin stopping target and the muons decay at rest. The signature of $\mu^{+} \rightarrow \mathrm{e}^{+} \gamma$ is a pair of a photon and a positron both carrying $52.8 \mathrm{MeV}$ energies, traveling to the back-to-back directions from the same position, created at the same time. Positrons are detected by the spectrometer consisting of the COBRA magnet, the drift chambers and the timing counters. Photons are detected by a liquid xenon (LXe) calorimeter located outside the magnet. The main background is the accidental pileup of a positron from the normal muon decay (Michel positron) and a photon either from the radiative muon decay (RMD) or the positron annihilation in flight (AIF). Another background is the RMD with neutrinos carrying little energy. The signal can be discriminated from the backgrounds with measuring the kinematic variables 


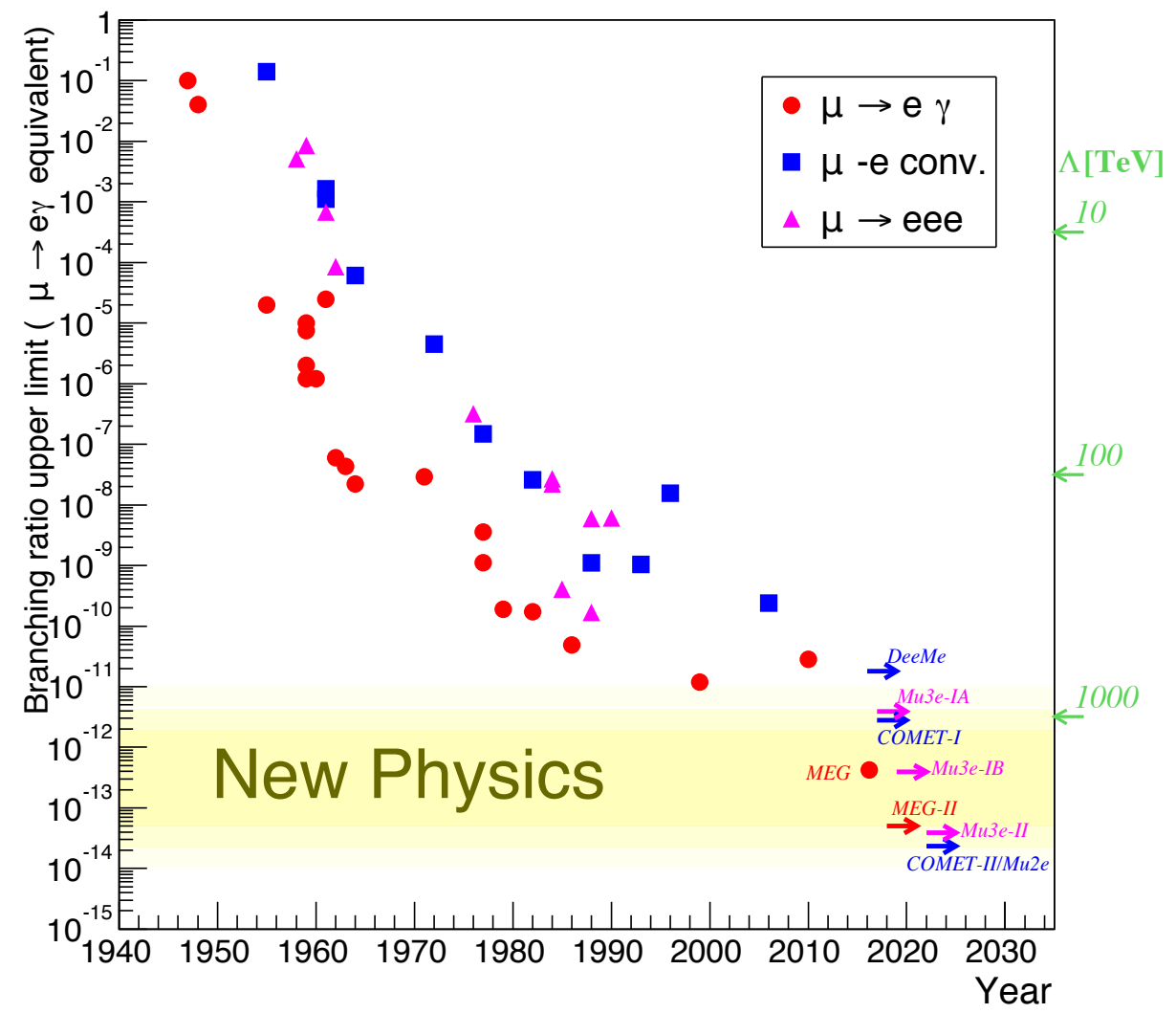

Figure 1: History of the $90 \%$ C.L. upper limits of muon cLFV channels. The upper limits are converted to $\mu \rightarrow$ e $\gamma$ equivalent values with assuming the dominant new physics contribution is the photonic dipole interaction. The right vertical axis show the new physics mass scale in the model independent parametrization [17]. This is based on a figure in [18].

(photon energy $E_{\gamma}$, positron energy $E_{\mathrm{e}}$, relative time $T_{\mathrm{e} \gamma}$ and relative polar and azimuthal angles $\left.\theta_{\mathrm{e} \gamma}, \phi_{\mathrm{e} \gamma}\right)$ precisely.

The physics data were taken in 2008-2013 (2008 data are not used because of an unstable detector condition). The previous result [19] based on the 2009-2011 data was published in 2013; no excess was observed and the expected and the observed upper limit at $90 \%$ C.L. was $7.7 \times$ $10^{-13}$ and $5.7 \times 10^{-13}$, respectively. The final analysis with the full data was done with improved positron track reconstruction algorithms, new event selection for rejecting very AIF-like photons and modified treatment of systematic uncertainties for the stopping-target in a robust way.

Figure 3 shows the four dimensional event distribution around the signal region. The background events distribute in the low energy region, and in the time-angle plane uniformly. There is not an apparent excess of events in the signal region shown by contours. The physics analysis was done with unbinned likelihood fitting on data in a wide region of kinematic variables to safely include a large part of the signal PDF. The stopping-target uncertainty, which is the dominant systematic uncertainty, was included as a nuisance parameter of the fitting. Table 3 shows the summary of the final results of the MEG experiment. No excess of events were found, and an upper limit of the branching ratio of the signal was set to be $4.2 \times 10^{-13}$ at $90 \%$ C.L. [9]. The obtained 

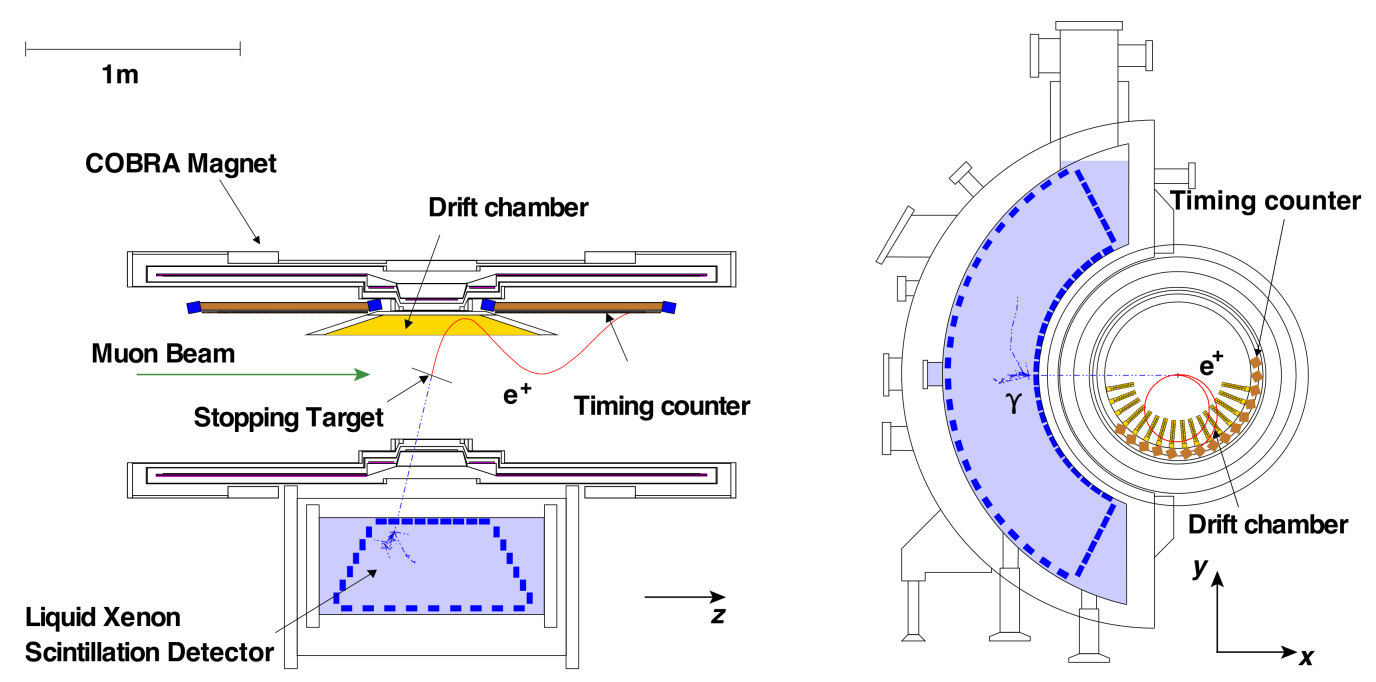

Figure 2: Schematic view of the MEG detector.

most stringent upper limit is 30 times lower than the limit given by the previous experiment [20].
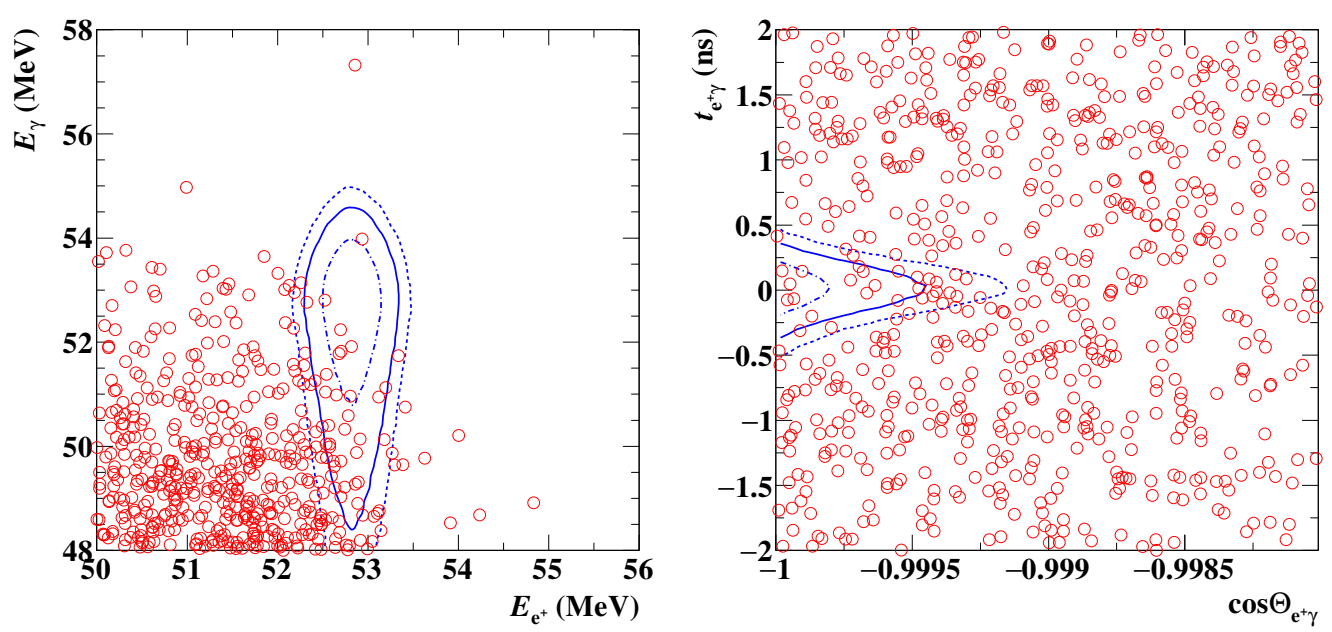

Figure 3: Event distributions in the analysis region of the MEG full data set. The energies of positrons and photons (left), the time and angle differences (right) are shown. Selections of $\cos \Theta_{\mathrm{e} \gamma}<-0.99963$ and $\left|T_{\mathrm{e} \gamma}\right|<0.24 \mathrm{nsec}$ are applied for the left plot, and $51.0<E_{\gamma}<55.5 \mathrm{MeV}$ and $52.4<E_{\mathrm{e}}<55.0 \mathrm{MeV}$ are applied for the right plot, where $\Theta_{\mathrm{e} \gamma}$ is the relative stereo angle between the photon and the flipped positron momentum vectors at the muon decay point. The contours show the signal PDF $(1 \sigma, 1.64 \sigma$ and $2 \sigma)$.

An upgrade of the MEG experiment is being prepared at PSI for aiming ten times higher $\left(4 \times 10^{-14}\right)$ sensitivity. For achieving the goal, a higher beam intensity, higher detection efficiency and a much lower background fraction are needed. More than two times higher muon beam is already available with the same beam line. For improving the resolutions and efficiency, all the sub-detectors are upgraded. The LXe photon detector is upgraded with replacing the two-inch photomultipliers (PMTs) on the photon-entering face with $12 \mathrm{~mm}^{2}$ VUV-sensitive silicon photo- 
Table 3: Best fits and 90\% C.L. upper limits of $\operatorname{Br}\left(\mu^{+} \rightarrow \mathrm{e}^{+} \gamma\right)$.

\begin{tabular}{|l|ccc|}
\hline dataset & $2009-2011$ & $2012-2013$ & $2009-2013$ \\
\hline Best fit $\times 10^{13}$ & -1.3 & -5.5 & -2.2 \\
\hline Expected upper limit $\times 10^{13}$ & 8.0 & 8.2 & 5.3 \\
\hline Observed upper limit $\times 10^{13}$ & 6.1 & 7.9 & 4.2 \\
\hline
\end{tabular}

multipliers (SiPMs). A new type of SiPM, which has a photo-detection efficiency larger than 15\% for LXe scintillation photons, was developed with Hamamatsu Photonics. The energy, position and time resolutions will be improved by the higher granularity and the uniform response over the photon-entering face. The positions of existing PMTs are further optimized for decreasing the energy leakage and for making the energy response uniform over the $\phi$ coordinate. A completely new drift chamber, which has a long single gas volume, will be used. The transparency of the signal positrons to the timing counters will be about two times higher than MEG due to the less material between the tracking volume and the timing counters. The tracking resolution will be much improved thanks to the better hit-resolutions and the increased number of hits. The new timing counter system consists of many small scintillation tiles coupled with $12 \mathrm{SiPMs}$ each. A signal positron hit $\sim 9$ tiles in average. By averaging the time measured by the multiple tiles, the time resolution will be $30-40$ psec. In addition to the upgrade of the existing sub-detectors, a new detector for identifying the background photons from the RMD will be added. For treating the increased event rate and the increased readout channels, a new TDAQ system based on the WaveDREAM board is being prepared. All the components are supposed to be ready in Autumn 2017 and physics data taking will follow an engineering run.

\subsection{Mu3e}

The Mu3e experiment is going to search for the cLFV decay $\mu^{+} \rightarrow \mathrm{e}^{+} \mathrm{e}^{-} \mathrm{e}^{+}$. The signal positron and electron from the three-body final state may have low momenta. The detector therefore needs to have high enough acceptance for the low momentum electrons and positrons. The internal conversion background events can be suppressed with using the momenta and the times of the detected particles. For reducing the accidental background events, matching of the vertex can also be used. Figure 4 shows the Mu3e detector for the phase I experiment. Silicon pixel detectors are put near and far from a conical muon-stopping target. The time of the signal electrons and positrons is measured precisely with a ToF system consisting of the scintillating fibers and tiles. With this detector design, the acceptance for the signal reaches about 70\%. A key technology is $50 \mu \mathrm{m}$ thick silicon detector embedding the readout logic and amplifiers (HV-MAPS). The TDAQ system to handle the 275 M HV-MAPS channels and $20 \mathrm{k} \mathrm{ToF}$ channels is also important for the experiment. The phase I experiment is supposed to be done in 2018-2020, the target sensitivity is better than $10^{-15}$, which is three orders of magnitude better than the current limit. For the phase II experiment, a new muon beam line with an intensity of $2 \times 10^{9}$ muons per second is needed. The detector will be upgraded with additional recurl stations for reducing the background rate. The phase II experiment will be later than 2020 aiming a sensitivity better than $10^{-16}$. 


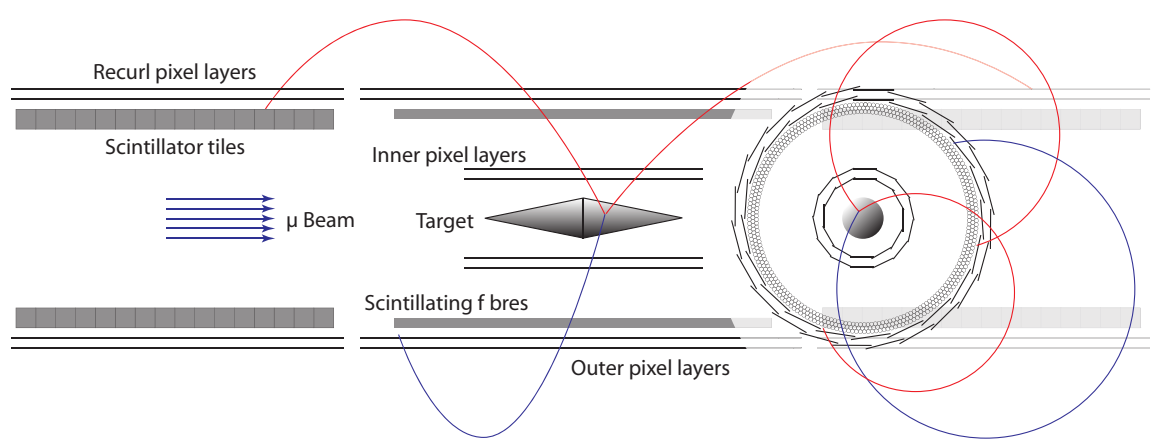

Figure 4: Schematic view of the Mu3e phase IB detector.

\section{$2.3 \mu \rightarrow$ e conversion experiments}

The signal of the $\mu \rightarrow \mathrm{e}$ conversion is a mono-energetic electron emitted from the bounded state of a muon in a nucleus. The backgrounds are from muon decays in orbit, cosmic rays and those correlated to the beam (muon and pion decay in flight, radiative pion capture, anti-proton induced backgrounds and other beam particles). While the dominant backgrounds of $\mu^{+} \rightarrow \mathrm{e}^{+} \gamma$ and $\mu^{+} \rightarrow \mathrm{e}^{+} \mathrm{e}^{-} \mathrm{e}^{+}$are accidental pileups of muon decays, there are no accidental background in $\mu \rightarrow \mathrm{e}$ conversion experiments. Muon beam with much higher instantaneous rate therefore can be used for $\mu \rightarrow \mathrm{e}$ conversion experiments. While the signal events have a long decay time due to the lifetime of the muon in nuclei, the major backgrounds coincide with the beam. The signal to background ratio can be much improved by using the delayed signal time window about 700 nsec. A pulsed muon beam with high instantaneous rate is therefore optimum for $\mu \rightarrow$ e experiments, while a DC beam is preferred by other muon cLFV decay experiments. The expected signal rate depends on the type of the new physics couplings (dipole, scalar or vector) and the target material. It is therefore possible to discriminate the new physics theories with measuring the signal rate with various target materials.

There are three future experiments, Mu2e at Fermilab in the U.S., COMET and DeeMe at J-PARC in Japan.

The DeeMe experiment is searching for the signal electrons emitted from the proton target. It will utilize the $3 \mathrm{GeV}$ proton beam at the MLF beamline in J-PARC. The experiment will be started in Japanese fiscal year 2016, which is much earlier than the other experiments. The expected sensitivity is $1.2 \times 10^{-13} \sim 2.1 \times 10^{-14}$.

Figure 5 shows the apparatus of the Mu2e experiment. Pions created in the proton target are extracted to an S-shape solenoid for eliminating photons and neutrons. A collimator is put in the solenoid to select low momentum negative muons. The muons from the pion decays are stopped on a target located inside the detector solenoid. The detector consisting with a tracker and a calorimeter detect only high momentum particles for reducing the occupancy. The detector is fully surrounded by cosmic-veto counters for rejecting the background. The construction of the experiment building 
was completed. The physics data taking is expected to start about 2021 aiming the single event sensitivity of $2.6 \times 10^{-17}$, which is four orders of magnitude better than the present limit.

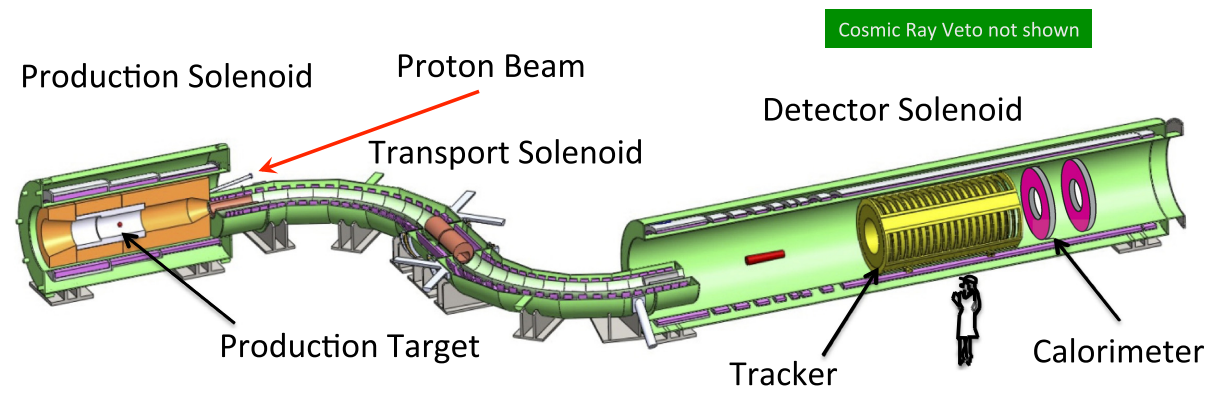

Figure 5: Mu2e setup.

Figure 6 shows the apparatus of the COMET phase II experiment. Pions are transported through a C-shaped solenoid and the muons are stopped on a target located inside a part of the solenoid. The detector is put after another solenoid to select the momentum of electrons. The group is taking a staging approach. In the phase I, the half of the pion transport solenoid is used, and the stopping target will be put inside the detector, which consists of a cylindrical drift chamber, trigger scintillation counters and Cerenkov detectors. Another detector based on straw tubes and LYSO crystals will be used for the beam background measurement after the physics data taking. The phase I experiment is supposed to be done in 2018 or 2019 with the expected single event sensitivity of $3.1 \times 10^{-15}$. The phase II will be started in about 2021 with the full apparatus. The expected single event sensitivity is $2.5 \times 10^{-17}$.

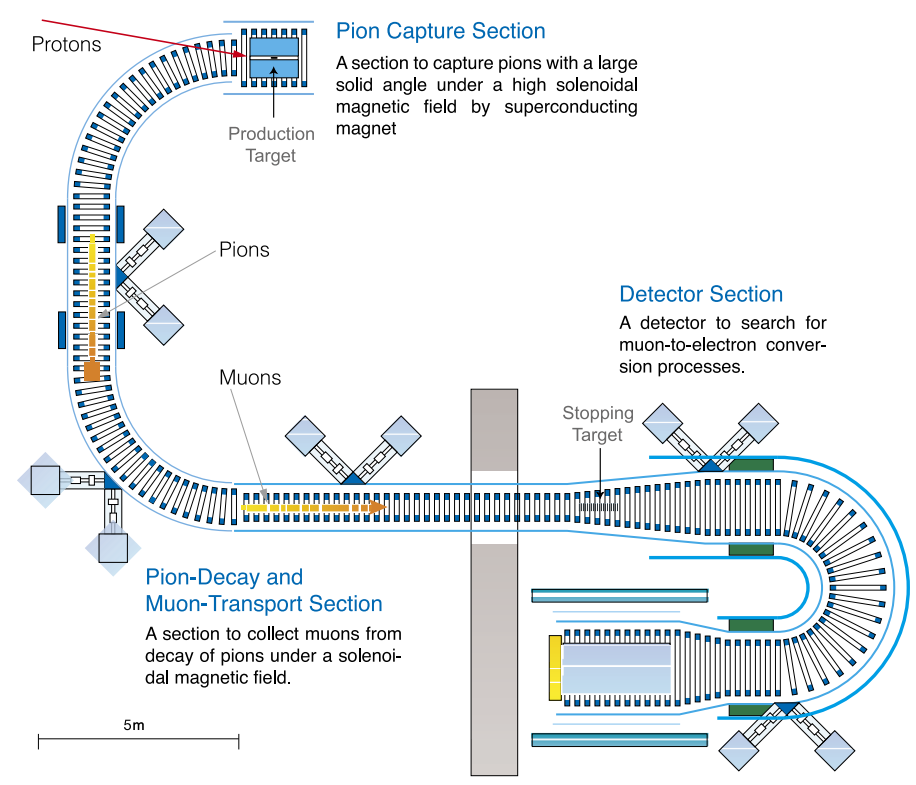

Figure 6: COMET Phase II setup. 


\section{$2.4 \tau$ lepton cLFV decay searches}

Rare decays of the $\tau$ lepton are searched for with $\tau$ pairs produced at B-factories and at the LHC. The analysis is done with tagging a $\tau$ decaying a normal way and using the other $\tau$ as a probe to search for rare decays. About fifty cLFV channels were analyzed by the Belle and BaBar experiments improving the previous results by CLEO for about two orders of magnitude. The many search channels, including photon+lepton, multiple leptons and hadrons, allow to cover the full spectrum of the new physics theories and to discriminate the new physics from the correlation between the channels. The sensitivity of the LHC experiments is in the same order as the BaBar and Belle experiments for some channels. None of the signal channels were discovered. The current limits are summaries in Fig. 7. The Belle II experiment will accumulate two order of magnitude more $\tau$ pairs than the previous generation B-factories by 2023-2024. The expected sensitivities of the most of the channels are two orders of magnitude better $\left(\mathrm{O}\left(10^{-10}\right)\right)$ while those of lepton+photon channels is in the order of $10^{-9}$ because of the higher level of the background.

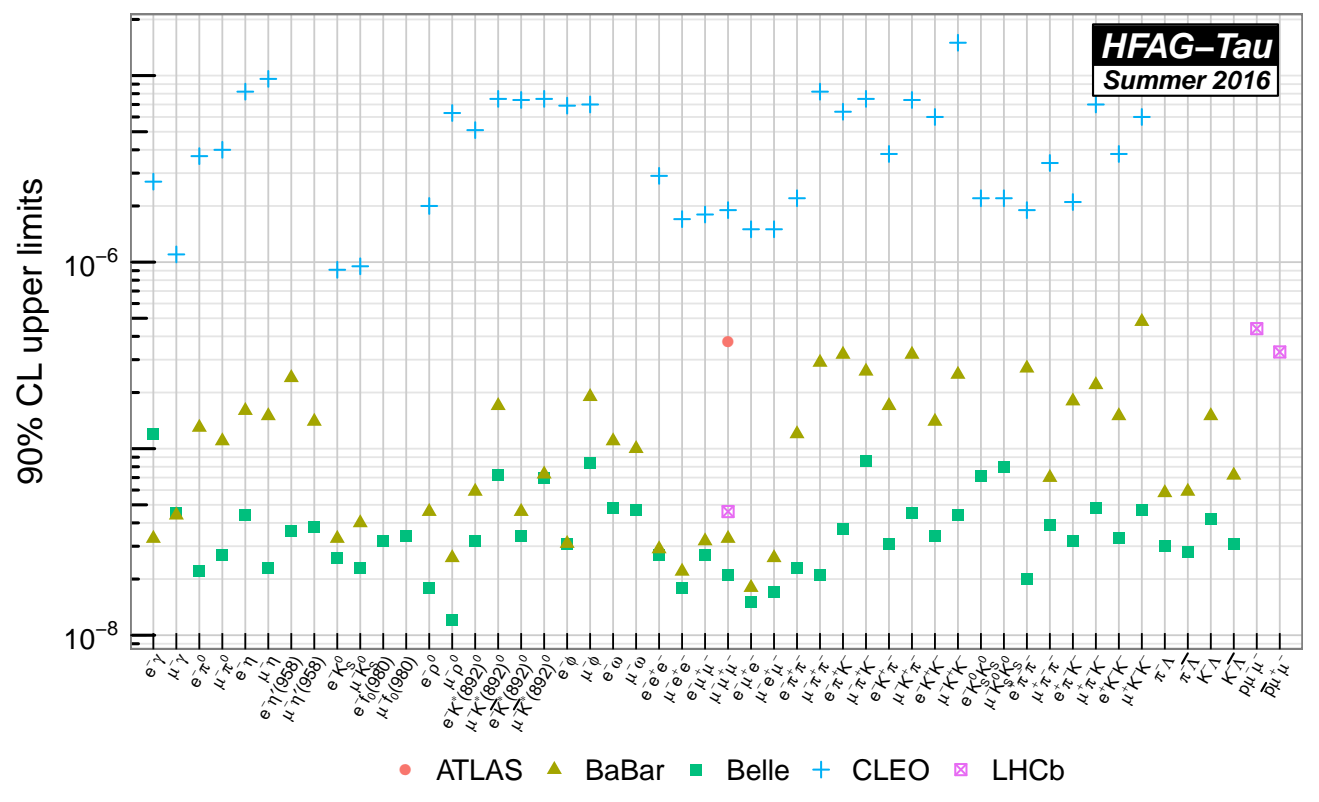

Figure 7: Current limits of $\tau$ LFV decays [21].

\section{Conclusions}

The new physics searches through indirect effects involving new particles are complementary to the direct searches in the high energy frontier experiments. The cLFV experiments have a higher reach of the new physics energy level. The most stringent and recent result was given by the MEG experiment $\left(\operatorname{Br}\left(\mu^{+} \rightarrow \mathrm{e}^{+} \gamma\right)<4.2 \times 10^{-13}\right.$ at $90 \%$ C.L. $)$ which constrains new physics models very strictly. There are plenty of future experiments and improved measurements planned, which will have up to four orders of magnitude higher sensitivity than the current limits. Once cLFV is observed, the combination of the several measurements would reveal the nature of the new physics; 
it is therefore important to realize all the planned experiments in the proposed schedule and with the aimed sensitivities.

\section{References}

[1] Muon (G-2) collaboration, G. W. Bennett, B. Bousquet, H. N. Brown, G. Bunce, R. Carey, P. Cushman et al., Final report of the E821 muon anomalous magnetic moment measurement at BNL, Phys. Rev. D 73 (2006) .

[2] A. Antognini, F. Nez, K. Schuhmann, F. D. Amaro, F. Biraben, J. M. R. Cardoso et al., Proton Structure from the Measurement of 2S-2P Transition Frequencies of Muonic Hydrogen, Science 339 (2013) 417-420.

[3] LHCB collaboration, R. Aaij, B. Adeva, M. Adinolfi, A. Affolder, Z. Ajaltouni, S. Akar et al., Measurement of the Ratio of Branching Fractions $\mathscr{B}\left(\bar{B}^{0} \rightarrow D^{*+} \tau^{-} \bar{v}_{\tau}\right) / \mathscr{B}\left(\bar{B}^{0} \rightarrow D^{*+} \mu^{-} \bar{v}_{\mu}\right)$, Phys. Rev. Lett. 115 (2015) 111803.

[4] LHCB collaboration, R. Aaij, B. Adeva, M. Adinolfi, A. Affolder, Z. Ajaltouni, S. Akar et al., Test of Lepton Universality Using $B^{+} \rightarrow K^{+} \ell^{+} \ell^{-}$Decays, Phys. Rev. Lett. 113 (2014) 151601.

[5] W. Altmannshofer and D. M. Straub, New physics in $b \rightarrow s$ transitions after LHC run 1, Eur. Phys. J. C 75 (2015) 382.

[6] S. Descotes-Genon, T. Hurth, J. Matias and J. Virto, Optimizing the basis of $B \rightarrow K^{*}$ ll observables in the full kinematic range, JHEP 05 (2013) 137, [1303. 5794].

[7] CMS collaboration, V. Khachatryan, A. Sirunyan, A. Tumasyan, W. Adam, T. Bergauer, M. Dragicevic et al., Search for lepton-flavour-violating decays of the Higgs boson, Phys. Lett. B 749 (2015) $337-362$.

[8] M. Blanke, A. J. Buras, B. Duling, S. Recksiegel and C. Tarantino, FCNC Processes in the Littlest Higgs Model with T-Parity: a 2009 Look, Acta Phys. Polon. B41 (2010) 657-683, [0 906.5454 ].

[9] MEG collaboration, A. M. Baldini, Y. Bao, E. Baracchini, C. Bemporad, F. Berg, M. Biasotti et al., Search for the lepton flavour violating decay $\mu^{+} \rightarrow \mathrm{e}^{+} \gamma$ with the full dataset of the MEG experiment, Eur. Phys. J. C 76 (2016) 434.

[10] SINDRUM collaboration, U. Bellgardt, G. Otter, R. Eichler, L. Felawka, C. Niebuhr, H. Walter et al., Search for the decay $\mu^{+} \rightarrow \mathrm{e}^{+} \mathrm{e}^{+} \mathrm{e}^{-}$, Nucl. Phys. B 299 (1988) $1-6$.

[11] SINDRUM II collaboration, W. Bertl, R. Engfer, E. Hermes, G. Kurz, T. Kozlowski, J. Kuth et al., A search for $\mu$-e conversion in muonic gold, Eur. Phys. J. C 47 (2006) 337-346.

[12] BABAR collaboration, B. Aubert, Y. Karyotakis, J. P. Lees, V. Poireau, E. Prencipe, X. Prudent et al., Searches for Lepton Flavor Violation in the Decays $\tau^{ \pm} \rightarrow e^{ \pm} \gamma$ and $\tau^{ \pm} \rightarrow \mu^{ \pm} \gamma$, Phys. Rev. Lett. 104 (2010) 021802.

[13] Belle collaboration, K. Hayasaka et al., Search for Lepton Flavor Violating $\tau$ Decays into Three Leptons with 719 Million Produced $\tau^{+} \tau^{-}$Pairs, Phys. Lett. B687 (2010) 139-143, [1 001.3221$]$.

[14] BNL E871 collaboration, D. Ambrose, C. Arroyo, M. Bachman, D. Connor, M. Eckhause, S. Graessle et al., New Limit on Muon and Electron Lepton Number Violation from $K_{L}^{0} \rightarrow \mu^{ \pm} e^{\mp}$ Decay, Phys. Rev. Lett. 81 (1998) 5734-5737.

[15] ATLAS collaboration, G. Aad et al., Search for the lepton flavor violating decay $Z \rightarrow e \mu$ in $p p$ collisions at $\sqrt{s}=8 \mathrm{TeV}$ with the ATLAS detector, Phys. Rev. D90 (2014) 072010, [1408.5774]. 
[16] CMS collaboration, Search for Lepton Flavour Violating Decays of the Higgs Boson in the $\mu$ - $\tau$ final state at $13 \mathrm{TeV}, \mathrm{CMS}$-PAS-HIG-16-005 (2016) .

[17] A. de Gouvêa and N. Saoulidou, Fermilab's Intensity Frontier, Annu. Rev. Nucl. Part. Sci. 60 (2010) 513-538.

[18] W. Ootani, An Experimental Review of Charged Lepton Flavor Violation in Muon Channel, J. Phys. Soc. Jpn. 85 (2016) 091002.

[19] MEG collaboration, J. Adam, X. Bai, A. M. Baldini, E. Baracchini, C. Bemporad, G. Boca et al., New Constraint on the Existence of the $\mu^{+} \rightarrow e^{+} \gamma$ Decay, Phys. Rev. Lett. 110 (2013) 201801.

[20] MEGA collaboration, M. L. Brooks, Y. K. Chen, M. D. Cooper, P. S. Cooper, M. Dzemidzic, A. Empl et al., New Limit for the Lepton-Family-Number Nonconserving Decay $\mu^{+} \rightarrow e^{+} \gamma$, Phys. Rev. Lett. 83 (1999) 1521-1524.

[21] http://www.slac.stanford.edu/xorg/hfag/tau/summer-2016/lfv-limits-plot.html, . 